\title{
Sub-keV Electron Gun Driven by Ultrafast THz Pulses
}

\author{
W. Ronny Huang ${ }^{1,2}$, Arya Fallahi' ${ }^{2}$, Xiaojun $\mathrm{Wu}^{2}$, Huseyin Cankaya ${ }^{2}$, Anne-Laure Calendron ${ }^{2}$, Koustuban \\ Ravi $^{1,2}$, Dongfang Zhang ${ }^{2}$, Emilio A. Nanni ${ }^{1}$, Kyung-Han Hong ${ }^{1}$, Franz X. Kärtner ${ }^{1,2,3}$ \\ ${ }^{\prime}$ Department of Electrical Engineering and Computer Science and Research Laboratory of Electronics, Massachusetts Institute of Technology, \\ Cambridge, MA 02139, USA \\ ${ }^{2}$ Center for Free-Electron Laser Science, Deutsches Elektronen-Synchrotron DESY, Notkestraße 85, 22607 Hamburg, Germany \\ ${ }^{3}$ Physics Department and The Hamburg Center for Ultrafast Imaging, University of Hamburg, Luruper Chaussee 149, 22761 Hamburg, \\ Germany \\ franz.kaertner@desy.de
}

\begin{abstract}
Strong-field, single-cycle THz fields accelerate electrons with peak energies of up to $0.8 \mathrm{keV}$ in an ultracompact $\mathrm{THz}$ gun with bunch charge of $45 \mathrm{fC}$. Energy spreads as low as $5.8 \%$ were also achieved.

OCIS codes: (320.0320) Ultrafast optics; (350.4990) Particles.
\end{abstract}

\section{Introduction}

Electron bunches delivered by ultrafast electron guns are essential for femtosecond electron diffraction and compact free electron lasers. Current hurdles facing the construction of conventional guns include the bulky, expensive high voltage feedthroughs or high power klystrons needed to power DC or RF guns, respectively, as well as the elaborate synchronization schemes needed for RF guns [1]. Further, both DC and RF guns have limitations in the accelerating field of about $10 \mathrm{MV} / \mathrm{m}$ and $200 \mathrm{MV} / \mathrm{m}$, respectively, due to breakdown mechanisms in common accelerator materials [2]. In accelerator physics, the electron beam size and bunch duration directly achieved from the gun is inversely proportional to the accelerating electric field. The need for more economical devices, coupled with the desire for higher field strength, has propelled the development of compact NIR- or THz-driven linear accelerator (linacs) structures with higher field breakdown thresholds [3,4] and intrinsic synchronization. The enormous potential advantages of photonic (NIR/THz) linacs, however, has not extended to photonic guns [5], even though the gun is quintessential to determining the final electron beam quality. Here we implement the first THz-driven electron gun based on a novel $\mu \mathrm{m}$-scale accelerating structure. We report electron acceleration from rest to almost 1 $\mathrm{keV}$ using a single-cycle THz pulse generated by a modest mJ-scale NIR laser. Quasi-monoenergetic energy spreads as low as $5.8 \%$ were achieved for $32 \mathrm{fC}$ bunches.

\section{Experimental setup}
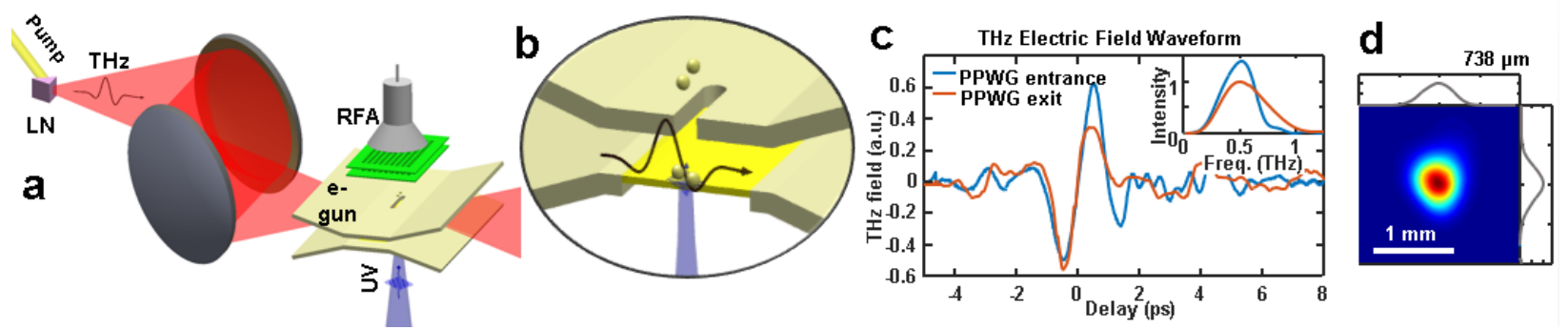

Figure 1: Schematic of setup (a) zoomed out and (b) cross-section zoomed in. LN: lithium niobate. RFA: retarding field analyzer. (c) THz electric waveform and spectrum (inset) at the entrance and exit of the PPWG gun structure. PPWG: parallel-plate waveguide. (d) THz intensity profile at free-space focus.

The THz gun, shown in in Fig. 1(a)-(b), takes the form of a copper PPWG with a thin-film copper photocathode (yellow) as the bottom plate, and an exit slit anode as the top plate. A THz beam is coupled into the TEM mode of the PPWG by the help of the angled taper. The PPWG spacing is $75 \mu \mathrm{m}$ (the TEM allows for subwavelength confinement). A UV pulse back-illuminates the photocathode, producing an electron bunch inside the PPWG by photoelectric emission. The bunch is then accelerated across the waveguide by the vertically polarized $\mathrm{THz}$ electric field. Then the bunch exits through the slit and is spectrally characterized by a retarding field analyzer (RFA) or counted by a Faraday cup. The THz pulses are generated by optical rectification in lithium niobate using the tilted pulse front technique. The max $\mathrm{THz}$ energy impinging the gun was $35.7 \mu \mathrm{J}$ after relaying off parabolic mirrors. Temporal waveform measurements at the gun "entrance" (at the focus of the parabolic mirror with the gun removed) and at the gun "exit" (at the focus of a second relay after the THz travels through the waveguide - not shown in schematic) reveals single-cycle temporal waveforms of about 1.2 ps FWHM (Fig. 1(c)). Taking into account the 
energy, waveform, and beam spot size (Fig. 1(d)), the focused THz has a calculated peak field in the range of 150 $\mathrm{MV} / \mathrm{m}$ in free space. Assuming the horizontal beam profile remains unaltered while the vertical profile is distributed uniformly across the $75 \mu \mathrm{m}$ spacing, and taking into account the coupling efficiency, the confined $\mathrm{THz}$ pulse inside the PPWG has a peak field in the range of $350 \mathrm{MV} / \mathrm{m}$.

\section{Results}

To determine the optimal emission phase, we record the kinetic energy spectra and bunch charge as a function of delay between the THz and UV pulses, as in Fig. 2(a)-(b). When the emission does not overlap the THz pulse (>2 ps or $<-2$ ps), there is minimal variation in electron energy gain with delay. When the emission overlaps the terahertz pulse (-2 to $2 \mathrm{ps}$ ), we observe a narrow energy spread with energy gain governed by the $\mathrm{THz}$ phase at which emission occurs. Between 0.3-0.8 ps, we observe suppression of photocurrent resulting from the fact that emission occurs at the positive half cycle of the THz field. Two delay positions were selected to be the operating points of the gun: the first delay, $\tau_{1}$, produced the highest electron energy gains while the second delay, $\tau_{2}$, produced the most monochromatic spectra. Fig. 2(c)-(d) shows the electron spectra at these two operating points for three $\mathrm{THz}$ energies. Each spectrum exhibits a unimodal distribution with a peak increasing monotonically with $\mathrm{THz}$ field. The spectral shape is asymmetric with a peak yield toward higher energies, followed by a sharp cutoff, and a pedestal toward lower energies. The high yield near the cutoff indicate that most of the electrons are emitted at the optimal $\mathrm{THz}$ phase and concurrently experience equivalent acceleration. The lower-energy pedestal can be attributed to the electrons which are emitted away from the optimal phase, resulting in relatively less energy gain. For maximal $\mathrm{THz}$ energy, we achieve a peak energy gain of $0.8 \mathrm{keV}$ at $\tau_{1}$. At $\tau_{2}$, we achieve an energy spread of $5.8 \%$ (with $0.4 \mathrm{keV}$ gain). The max bunch charge was measured to be $40 \mathrm{fC}$ and $32 \mathrm{fC}$ at $\tau_{1}$ and $\tau_{2}$, respectively.
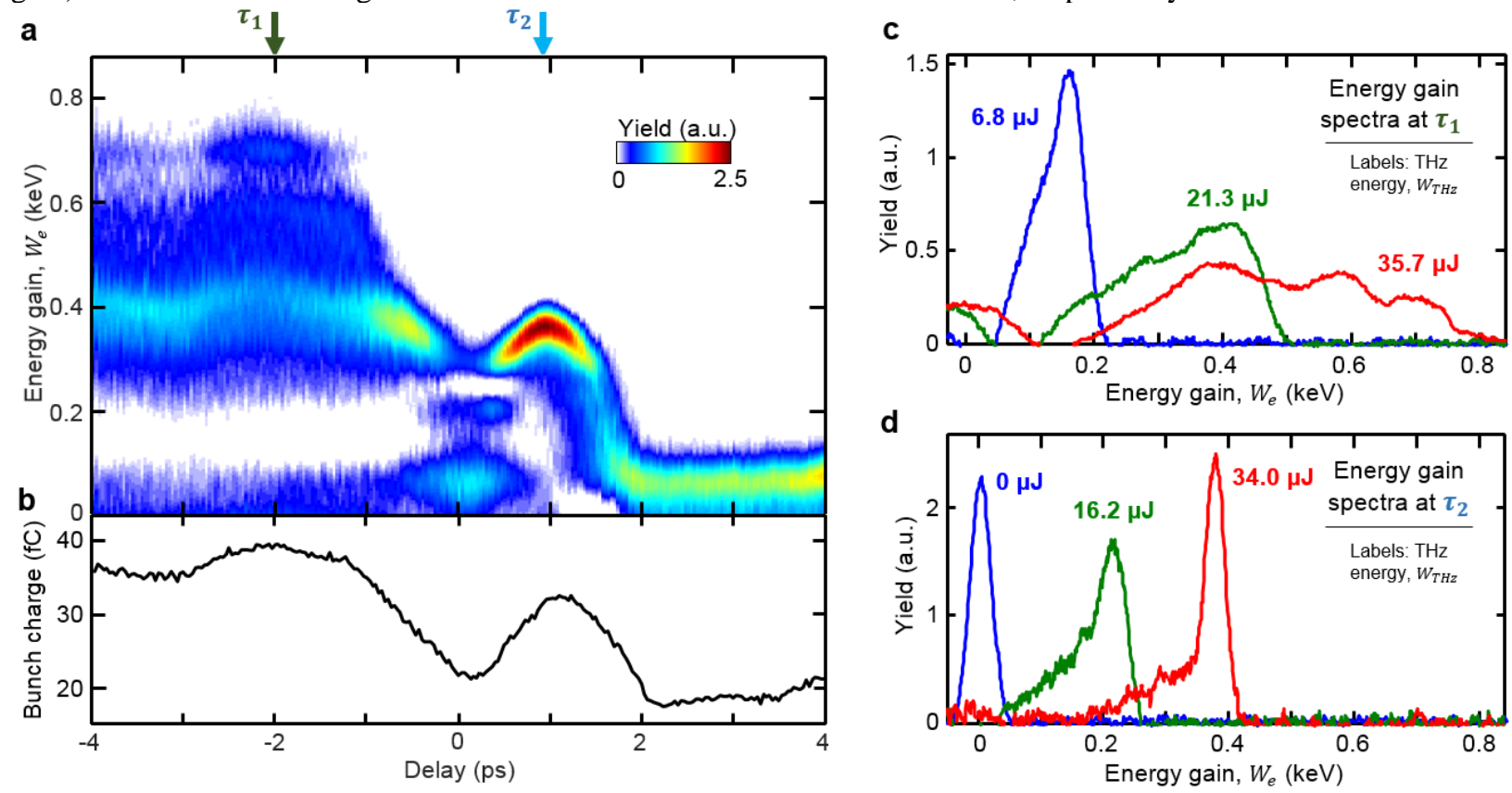

Figure 2: (a)-(b) Spectrogram showing (a) energy gain spectra and (b) bunch charge as function of delay between $\mathrm{UV}$ and $\mathrm{THz}$, at $\mathrm{max} \mathrm{THz}$ energy. (c)-(d) Accelerated electron spectra at delay (c) $\tau_{1}$ and (d) $\tau_{2}$ for several THz energies.

By plotting the spectra as a function of THz energy as shown in Fig. 3(a)-(d), we observe that the energy gain scales linearly as THz energy, or equivalently, as the THz field squared. This can be explained by $W_{e}=p_{e}^{2} / 2 m \propto E_{\mathrm{THz}}^{2}$, where $W_{e}$ is the electron energy gain, $p_{e}$ the electron momentum gain, and $E_{\text {thz }}$ the THz field. We also observe that the relative energy gain is constant with THz energy at $\tau_{1}$ while it is monotonically decreasing with $\mathrm{THz}$ energy at $\tau_{2}$ as in Fig. 2(e)-(f). Furthermore, the bunch charge is monotonically increasing at $\tau_{1}$ and plateaus after $7 \mu \mathrm{J}$ at $\tau_{2}$ as in Fig. 2(g)-(h). The plateau in the the bunch charge indicates that the THz field overcomes the space charge force and extracts all the emitted electrons [6] 

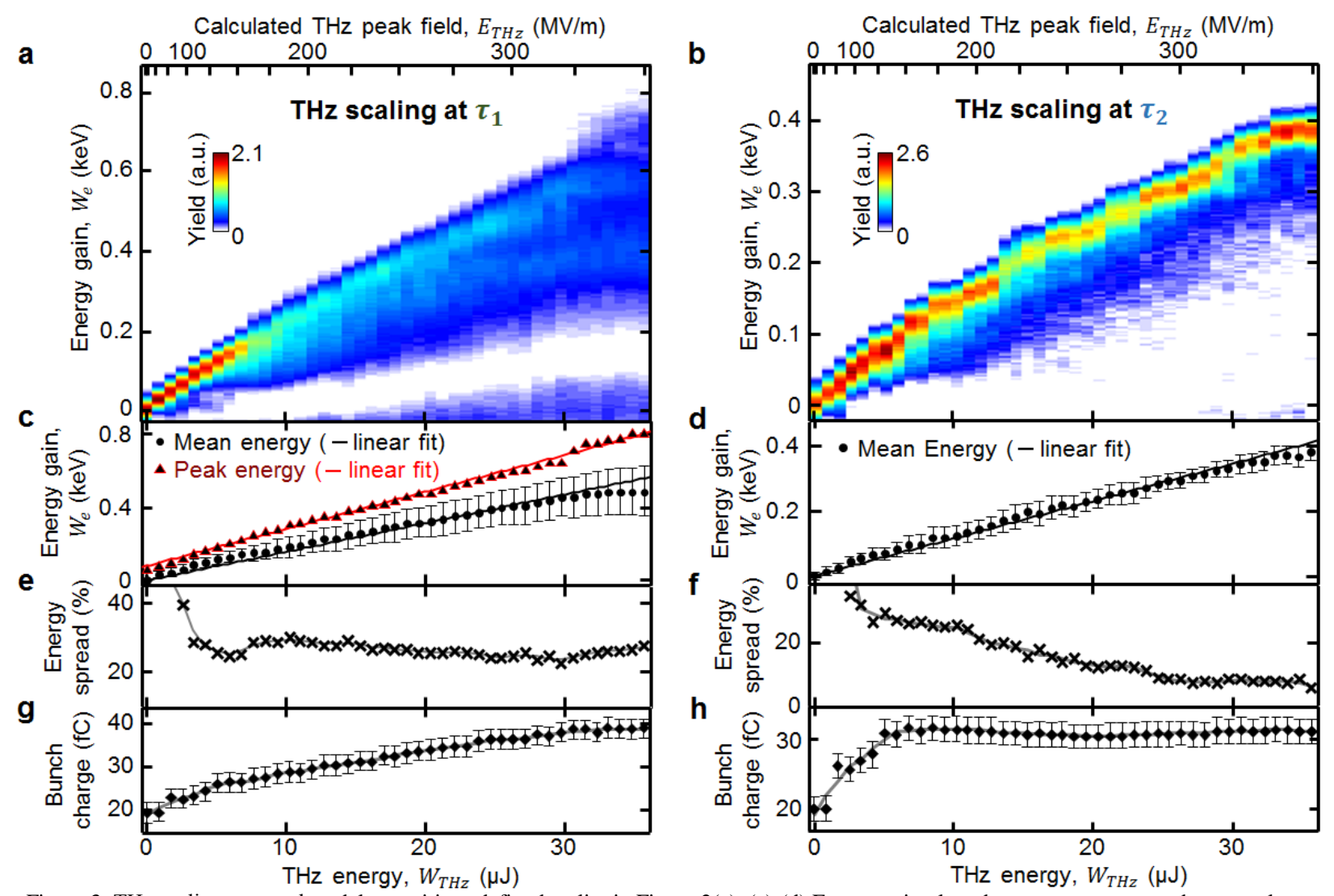

Figure 3: THz scaling at $\tau_{1}$ and $\tau_{2}$, delay positions defined earlier in Figure 2(a). (a)-(d) Energy gain plotted on a spectrogram and scatter plot to highlight its scaling as a function of accelerating THz energy or THz field. Error bar radius is equal to the absolute RMS energy spread. (e)-(f)

Percent RMS spread of the accelerated bunch. $(\mathrm{g})-(\mathrm{h})$ Total detected bunch charge exiting the gun. Error bar radius is equal to the RMS instrument noise

\section{Conclusion}

We have built a first electron gun driven by strong THz fields, with close to $1 \mathrm{keV}$ acceleration. The robust gun was operated over 1 billion shots without noticeable degradation.

\section{References}

1. G. J. H. Brussaard et al., "Direct measurement of synchronization between femtosecond laser pulses and a $3 \mathrm{GHz}$ radio frequency electric field inside a resonant cavity," Appl. Phys. Lett. 103, 23 (2013).

2. B. Spataro et al., "Technological issues and high gradient test results on X-band molybdenum accelerating structures," Nucl. Instruments Methods Phys. Res. A 657, 114-121 (2011).

3. K. J. Leedle, R. Fabian Pease, R. L. Byer, and J. S. Harris, "Laser acceleration and deflection of $96.3 \mathrm{keV}$ electrons with a silicon dielectric structure," Optica 2, 158 (2015).

4. E.A. Nanni et al., "Terahertz-driven linear electron acceleration," Nature Communications 6, 8486 (2015).

5. W. R. Huang et al., "Toward a terahertz-driven electron gun," Scientific Reports 5, 14899 (2015).

6. J. Rosenzweig et al., "Initial measurements of the UCLA rf photoinjector," Nucl. Instruments Methods Phys. Res. Sect. A Accel. Spectrometers, Detect. Assoc. Equip. 341, 379-385 (1994). 